\title{
Doorbell Automation using AI and IoT for old Aged and Differently Physically Challenged People
}

\author{
Anmol Singh ${ }^{1}$, AnuragSinha ${ }^{2}$ \\ ${ }^{1}$ Department of Computer Science and Engineering, Research scholar, Amity University Jharkhand \\ Ranchi, Jharkhand, India \\ ${ }^{2}$ Department of Information Technology, Research scholar, Amity University Jharkhand \\ Ranchi, Jharkhand, India \\ Email: anmolsingh15499@gmail.com ${ }^{1}$ anuragsinha257@ gmail.com²
}

\begin{abstract}
In this paper, I propose a smart door and doorbell system for home automation using the Internet of things (IoT) and Artificial Intelligence (AI) for old aged and differently able people to make their life easy and secure. In this model, I am using Raspberry Pi for managing various interfaces and communications. The model consists of multiple interfaces, including sensors, actuators and multiple inputs and out interfaces. To implement my IoT solution I have used AWS (Amazon web service) IoT to provide many cloud services to support IoT-based applications. The apps give access to IoT devices and the features the cloud services offer to those connected. The tools use various technologies and protocols such as Wi-Fi or cellular data to communicate with the cloud. The mode of communication is via MQTT (Message Queuing and Telemetry Transport) process. I have also used various software, including device software, control services, data derives and core services provided by the AWS IoT, making this model efficient and scalable. The overall aim of this paper is to make appropriate use of the technology for the needy and coming generations to come
\end{abstract}

\section{Keywords-Smart Doorbell System, Raspberry pi, MQTT, AWS IoT}

\section{INTRODUCTION}

Today, about 962 million of the global population is aged 60 and above and $10 \%$ of the world's population is differentlyabled people as predicted by WHO. Nearly in all families, some old aged people need care with time. Their mobility decreases with age, but they want to live the same way they used to live. Another section of people is the differentlyabled people who have difficulty in speaking, moving, seeing, or hearing things around. When an intruder rings the bell, they often panic or want to open the door, but it takes time. It's a matter of concern for their family members to leave them alone in their homes due to the above reason and for security issues. Someone needs to be around them. This gives rise to the feeling of inferiority

Advancements in technology such as Artificial intelligence and the Internet of Things, which has a vital role in home automation, are now making life more comfortable. But no such IoT projects have yet been developed specifically for the above group of people. Thus, in this paper, I am proposing a theoretical model that can solve this problem when implemented practically. In this paper, I am using Raspberry Pi as an application platform as it is widely used in recent years; it also provides comfortable use support and documentation. Raspberry Pi is connected to internet access, such as Wi-Fi. It uses python programming to connect and communicate with the amazon Web Service Internet of Things (AWS IoT ) as AWS allows Bi-directional communication between the devices connected to Internet, embedded devices and various AWS IoT such as cloud servers, database, analytics. Raspberry $\mathrm{Pi}$ is also connected to 3 sensors, which are two motion sensors and a one-touch sensor. It has a bell that rings when an intruder or member rings the doorbell. I have used AWS IoT Device SDK as a software kit that connects hardware devices or mobile applications, allowing devices to connect, authenticate and synchronize messages via IoT using the MQTT process. Face recognition Machine Learning models can be installed with the help of AWS IoT Greengrass ML inference on Raspberry Pi, which can perform face recognition locally.

\section{LITERATURE REVIEW}

Antunes and Grilo [1] described a web-based intrusion alarm system. It works on developing control and monitoring hardware of the distributed web-based intrusion alarm system. The alarm system should have at least one control panel, rechargeable battery, backup power supply and internal or external keypad, plush interior and a surrounding intrusion detector and an external siren.

In [2] I saw a short message service (SMS) intrusion alarm system for motorcycles. This guy is Owners of a potential intrusion into motorcycles via alarm and SMS (Short Message Service) PIC microcontroller programming. Research work included the PIC16F877A microcontroller interface. With a nokia6100 mobile phone connected in simple dual mode. 
From [3], the authors studied the security and privacy issues of the IoT.Four perspectives. First, they point out the limitations of applying security to IoT devices (e.g., Battery life, computer Power) and the proposed solution (e.g. Light Encryption schemes designed for embedded systems). They summarize the classification of IoT attacks (e.g., physical, remote, Area, etc.). Thirdly, they focus on mechanisms and architecture. Designed and implemented for authentication and authorization purpose. Finally, analyze security issues at multiple levels. (Ex. Physical, network, etc.).

The authors of [4] also demonstrated the possibility of realtime remote monitoring via IoT-based doorbells. All of these reveal endless applications for IoT-based intelligent security systems. Security systems are often breached by smart thieves, requiring deterrence measures to keep up with the criminals.

Intelligent Transport systems that use visual motion sensors Proposed by M. Soga and K.Yamada [5]. Andrew Burkett has come up with an excellent plan. Connect the Raspberry Pi to the base of the 6-axis gyro sensor And on the MPU6050 chip. Moreover, Installing the sensor interface and SMBus module with Simple Python code to read the data Proposal [6].

\section{Data Collection and its Significance}

Finding the problem faced by the old-aged and differentlyabled people, I have taken an online survey which justifies the use of technology such as IoT and AI for this section of the society can become a problem solving and make their lives easier

\section{Do you have any old aged person in your family or you are in contact with?}

60 responses
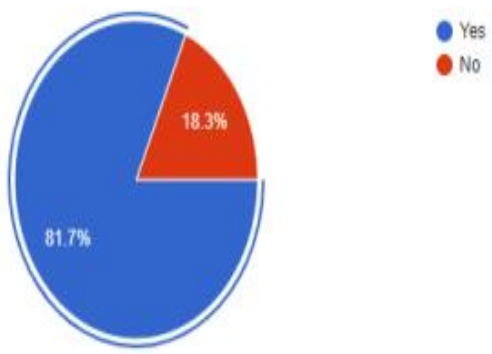

With 60 responses, $81.7 \%$ of people contact old aged, and $45 \%$ contact differently-abled people. Out of which $73.3 \%$ have a problem when they are alone in their home.

Do they have problem in opening and closing the door?

60 responses

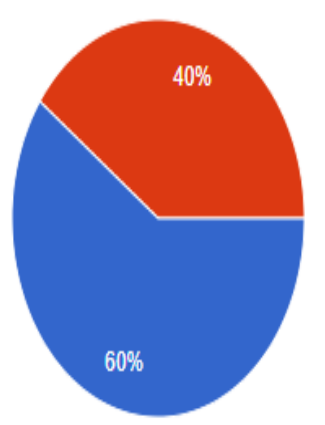

Do they face problem when they are alone in their room or house?

60 responses

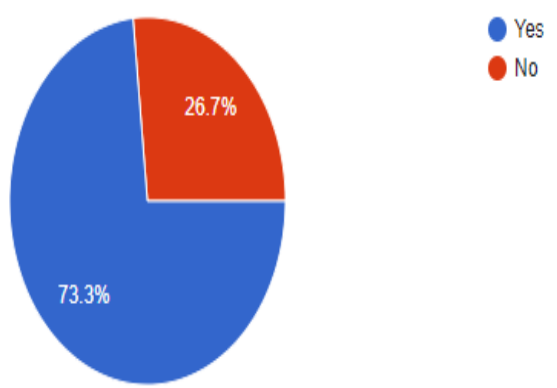


Do they panic when an intruder rings the doorbell when no one else is in the house? 60 responses

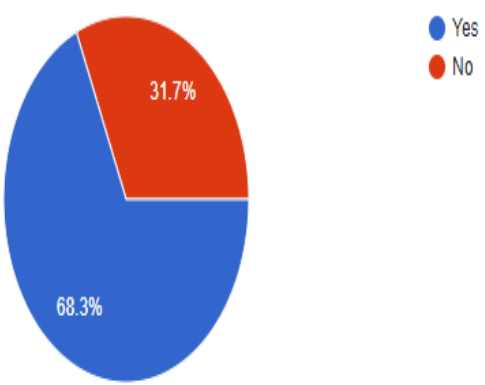

$73.3 \%$ of security and safety is the primary concern, and $83.3 \%$ agreed that doorbell automation could solve the old aged and differently-abled people's above problems.

From the above analysis, I conclude that technologies like IoT and AI can solve the problems faced by this section of society. Thus, in the next section, I propose a theoretical model that can efficiently solve these security problems when implemented.

\section{Proposed Model Architecture}

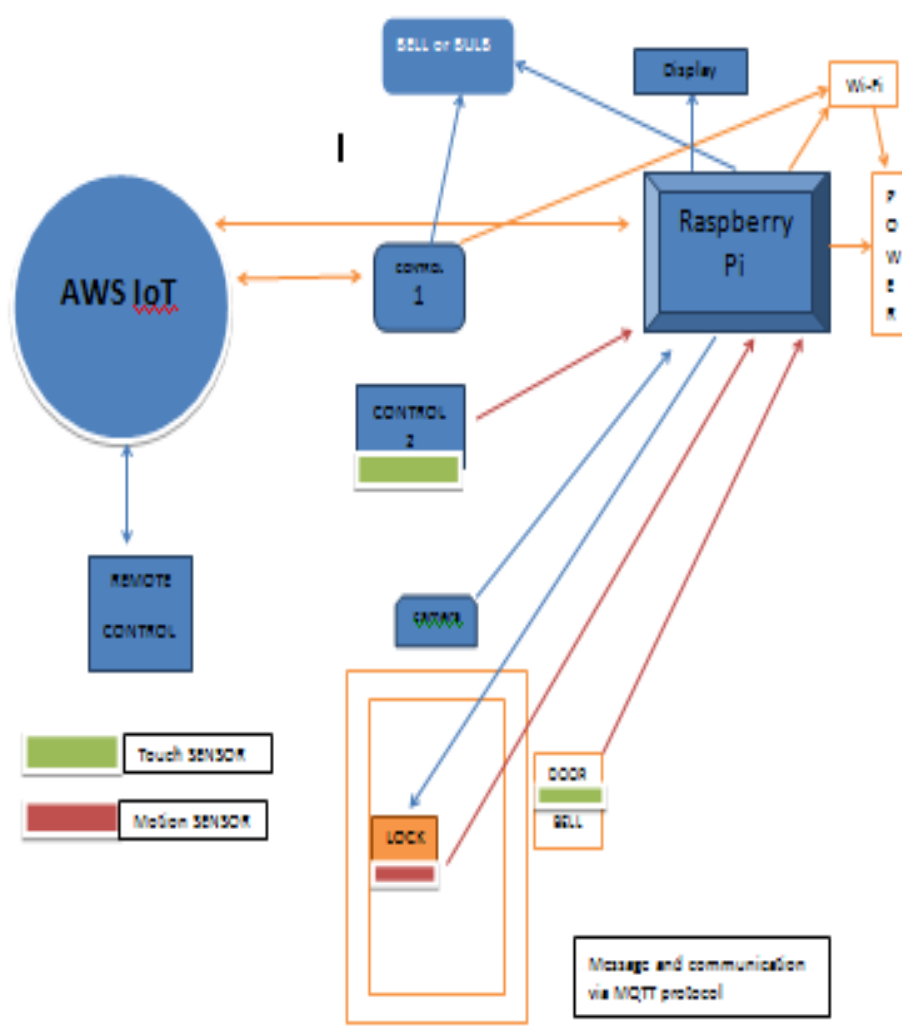

Figure 1: Main Model

Vol. 4 (4), October 2020, www.ijirase.com

\section{Model Description}

I am using AWS IoT as it is the only cloud service that integrates data management and rich analysis of data which is easy to use with its multi-layered security and superior AI integration AWS IoT, which has been proven a secure IoT cloud platform. I also chose this cloud platform as it is scalable. It offers different software and services like Free RTOS and AWS IoT Green grass, a microcontroller OS that makes low-power devices easy to deploy and manages local messaging, synchronizing, and machine learning inference. The connecting and control service like AWS IoT Core, AWS IoT device defender and AWS IoT device management allows secure connection with the cloud, audit and monitor remotely. Analytics services like AWS IoT analytics, AWS IoT Sidewise, AWS IoT events, AWS IoT thing graph operates on the data produced by the things and extract data from the IoT data stored in the cloud.

\section{Mosquitto Broker}

I have used Mosquitto broker as a lightweight open-source message broker written in $\mathrm{C}$ language. It has a good collection of libraries to implement MQTT, suitable for lowpower computer board computers like Raspberry Pi to full servers like AWS IoT. In this model, I am using this broker for two-way communication between Raspberry Pi and AWS IoT.

\section{Python}

With a clean syntax and robust programming, python programming connects my project to the real world. Python comes pre-installed on Raspbian and the Raspberry pi foundation mainly selected this language as the primary language because of its versatile nature and ease of use. It can be used to read input from the physical sensors connected to Raspberry $\mathrm{Pi}$ and send output to an external component using Python scripts. Simultaneously, AWS IoT Device SDK for Python enables developers to create and write python scripts that will allow their devices to access the AWS IoT cloud via MQTT.

\section{The IoT things connected to AWS IoT are:}

1) Raspberry Pi 4 -It is the latest low-cost Raspberry pi computer that has Raspbian OS. It is connected to various devices called 'things' and 'Internet Gateway' So the sensors connected to raspberry pi provide things with the Internet Gateway.

Raspberry pi 4 has a micro SD card slot for loading the Raspbian operating system like other Raspberry computers. Pi 4 models have a Broadcom BCM2711, quad-core Cortex- 
A72 (ARM v8) 64-bit SoC at $1.5 \mathrm{GHz}$.Memory is 4GB and 40pins - GPIO headers.

2) Motion Sensor- The motion sensor is PIR Motion Sensor placed under the door lock. It senses human/object when approaches it at a specific distance and activates the required task.

3)Motor sensor-Motor sensor is a Servo motor, which acts as a door lock. It rotates to a specific rotation angle when PWM (pulse width modulation) signals are sent to the motor.

4)Touch sensor -There are two touch sensors: the Momentary capacitive touch sensor, which is used as Doorbell and Control 2, which will activate as long as someone is touching it. Control 2 will unlock the door from inside.

5) Camera - The Raspberry Pi Camera Module v2 has Sony IMX219 8-megapixel sensor that spectator humans via video or image.

6) Control 1 \& Remote control- Here, control 1 can be a remote or an Android application with AWS IoT mobile SDK installed, which controls the door's opening and closing. The remote control can be an Android app that will notify the family members when an intruder enters the house.

7) Bell - Here, a HiFi Berry DAC HAT + ADC for audio and speaker can be installed, which will act as an alarm that will notify the people inside the house. DAC+ADC provides perfect audio production by which we can record and play an analog input.

8) Display- It can be a small display screen LCD that can be used to Espectate the outside the door.

\section{Configuration and Implementation}

\section{Configuration}

Raspbian OS is being installed in Raspberry Pi 4 computers. All the input and output units are PIR motion sensor, Servo motor,Raspberry Pi Camera Module v2, Momentary capacitive touch sensor, One buttoned remote control, LCD screen, and HiFi Berry DAC HAT + ADC installed with speaker and microphone are being configured. The sensors are being connected through the GPIO pins of the Raspberry Pi. A fully secured door lock is built and the safety is performed by servo motor. The next step is to log in with an existing or new account to AWS official website. Then navigate to IoT core. All the devices are registered to the AWS IoT core. Certificates are downloaded and installed and policy is created. Mosquito MQTT is bridged with Raspberry Pi and AWS IoT. There is a standard android app developed for the client. All the necessary python scripts are being created.

\section{Implementation}

When an intruder stands in front of the door for more than 2 minutes or when someone rings the doorbell, the sensors gets activated. The camera captures the photo/video. The Raspberry pi gets notified and rings the doorbell and display the photo/video to the people inside the house. Raspberry pi is connected to $\mathrm{Wi}-\mathrm{Fi}$, collects the data and sends it to the broker. The AWS IoT further receives the broker's data and sends an alert message to the people who are not in their home via the android app notifying the presence of an intruder in their home. Raspberry pi further receives the relevant information from the central control via AWS IoT. The remote control gets activated and the people in the house can open the door from 1 press of a button.

The key model is shown in Fig1 because the customer wants it. It can be changed and it is possible to delete certain components and render sub-models.

- For old aged people who don't live with their family will not require an android app.

- People who have a problem in hearing may not need a microphone and bell or speaker. External vibrators can be installed with the Raspberry pi to feel the presence of an intruder.

- For people who cannot see, LCD would not be required.

\section{Working Mechanism}

This model works on the MQTT (Message Queuing Telemetry Transport) protocol for the exchange of data between clients and servers. I have used MQTT because it is a light weight messaging protocol that uses TLS (Transport Layer Security) encryption. It is a real-time low power and network usage messaging protocol. The standard MQTT is a broker based protocol. 


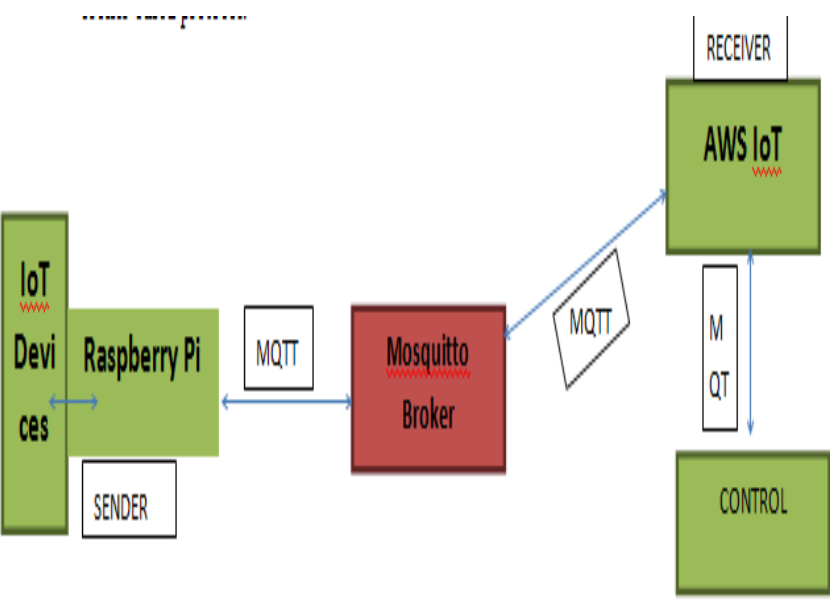

Figure 2: Working model

From fig 2., when an intruder stands outside the door or rings the doorbell, the various sensors sense their presence and send signals electrical signals to the Raspberry pi (rings the doorbell). The Raspberry pi is the sender cloud that publishes this data from the various IoT devices (such as doorbell and door lock sensors) of a particular IP address/client ID to the Mosquito broker. The broker breaks the message into topics.AWS IoT being the receiver cloud, has already subscribed to specific issues and receives messages from the broker. AWS IoT then notifies the controller (mobile and remote control) of the relevant and required message received from the broker, i.e., intruders in front of the door. Then the controller performs the necessary action and notifies back to the AWS IoT. This time cloud then publishes the related messages in the form of topics back to the Mosquito broker. Raspberry pi further subscribes to those topics and receive information and perform the final task (opening and closing the door).

AWS IoT also offers AWS IoT Green grass, which includes pre-built Amazon Sage Maker for devices like raspberry pi so we need not build or train machine learning models from scratch. AWS IoT Green grass offers ML inferences locally on the IoT devices. AWS IoT Green grass also provides pretrained Machine Learning models of Amazon Sage Maker, which we can directly deploy to the cloud. The inferences can be performed locally on devices, which reduces the cost of sending IoT device data back to the cloud for machine learning inferences. It can detect the outliner and send the data to the cloud and further; Amazon Sage Maker can reclassify and restrain and improve machine learning models.

\section{Advantages and Limitations}

Advantages:

- $\quad$ The above model can make life easier and efficient for the old-aged and differently challenged people.

- Increases security and safety for the client.

- Raspberry pi being of low cost and massive processing power, can support multiple interfaces simultaneously, making it easy to build applications as it supports languages such as Python and Linux.

- $\quad$ MQTT provides Quality of Service Levels (QoS) and can carry any data. It uses low sized packets and can be used for low bandwidth applications with low power consumption.

- Easy monitoring, controlling, managing, adding devices and data from and to AWS IoT cloud service with best security practices is provided at a scalable price, i.e., we pay less for the best we get.

- Controlling the entire system is easy and takes no time to learn for the client.

\section{Limitations :}

- It can create difficulty for blind people to adapt to the system compared to other people.

- The platform sets AWS IoT services limits. There is a technical support fee with general cloud computing issues.

- There are heating issues with the high performance and processing speed of Raspberry Pi.

- $\quad$ MQTT operates over TCP and it often affects longterm battery consumption. As networks grow large, brokers can affect scalability.

- Data transfer totally depends on Wi-Fi.

- This model, if implemented for ordinary people, can make them lazy.

\section{Conclusion}

The paper is based on the design and implementation of Door and Doorbell automation. This is an entirely sensorbased IoT model. This model can help the old aged people and differently-abled people in various aspects. With the help of a single button, they can open the door when someone rings the doorbell and they get notified by their presence. At the same time, in their android app, household members who are not with them are alerted through the cloud when the door is opened or when someone rings the doorbell. This model can be modified as per the need and requirement of the client. For example, a bulb can be placed for the people who have a problem in listening so that they can get notified of the presence of an intruder by the blinking of the bulb. For others who have a problem in seeing get notified via a small microphone and speaker, which gets activated when someone rings the doorbell. Others can easily via a small LCD screen placed inside the house. This model is secure as all the data are being stored 
in the cloud. One can access it at any time. Thus the above model is cheap concerning the functionalities it provides to the client.

This can become a life-changing tool for the needy and with its demand after testing and manufacture, it can become a source of employment for many people worldwide. In the future sub, models can also be developed for ordinary people for everyday use.

\section{References}

[1] Autunes, R.M., and Grilo, F.L. (2009) unwelcome person Alarm System: The Road Ahead. Advanced Technologies, Kankesu Jayanthakumaran (Ed.), ISBN: 978953-307-009-4, InTech.

[2] Rafiz, M. (2010) "Intruder Alert System via SMS for Motorcycle". unpublished Thesis work, University of Malaysia

[3] Yang Y, Wu L, Yin G, Li L, Zhao H . A survey on security and privacy problems in internet-of-things. IEEE net Things J 2017 .

[4] Ping W, Guichu W, Wenbin X, et al. (2010) Remote Monitoring Intelligent System supported Fingerprint Door Lock, International Conference on Intelligent Computation Technology and Automation.

[5] Yamada, K. and M. Soga, A compact integrated visual motion device for ITS applications. Intelligent Transportation Systems, IEEE Transactions on, 2003.

[6] Azrina Abd Aziz, Y. Ahmet S,ekercioglu, Paul Fitzpatrick, and Milosh Ivanovich, "A Survey on Distributed Topology Control Techniques for Extending the period of Battery power-driven Wireless device Networks", IEEE communications surveys \& tutorials, VOL. 15, NO. 1, halfmoon 2013. 